\title{
The reconstruction of static visual forms from sparse dotted samples
}

\author{
WILLIAM R. UTTAL \\ Naval Ocean Systems Center, Kailua, Hawaii \\ NANCY S. DAVIS and CYNTHIA WELKE \\ SEACO, Kailua, Hawaii \\ and \\ RAMAKRISHNA KAKARALA \\ The University of Michigan, Ann Arbor, Michigan
}

\begin{abstract}
This study explored the ability of observers to recognize very sparsely sampled, stereoscopic, dotted surfaces. The observers' performance exceeded that of a simple surface-fitting algorithm that served as a first approximation model to the solution of the perceptual problem. We explored various attributes of the stimulus surfaces, but were unable to find any single attribute that could account for the measured performance. Therefore, we propose that the observers used several global surface attributes jointly to distinguish one form from another. Collectively, these attributes define what is meant by form in this study. We suggest that our results argue for a globally precedent, multidimensional model of form reconstruction as opposed to a local-feature, unidimensional interpretation.
\end{abstract}

How do observers reconstruct percepts from abstractions or caricatures that only sparsely sample a stimulus object? For many decades it has been quite clear that the human visual system has a powerful ability to fill in, close, and perceptually complete partial stimuli. Examples of completion phenomena are ubiquitous in geometric illusions, in the work of the Gestalt psychologists (as discussed, for example, in the work of Wertheimer, 1923, and Kohler, 1929/1947) and in many reports by contemporary perceptual researchers. Modern artists also take advantage of this ability to perceptually complete by suggesting complex images with simple abstractions of reality rather than by photographic reproduction of the full details of a scene.

The ability to reconstruct images is so powerful that it is often difficult to convince an observer that an object that was perceived was not, in fact, present. In one wellknown example, a carefully designed demonstration is required to convince an observer that each retina possesses a $5^{\circ}$-wide lacuna. Pursuing another proof of our reconstructive abilities, Townsend and Ashby (1982) showed that observers viewing stimuli that were close approxi-

\footnotetext{
This research project was supported by the Office of Naval Research under work request N0001485WR24281. The scientific officer for this project is John J. O'Hare. The authors express their appreciation to the following colleagues at NOSC-Hawaii who consulted with us in the preparation of this report: Barry Kishimoto, John E. Sigurdson, E. Hugh Spain, and Robert E. Cole. Reprint requests for this report should be addressed to William R. Uttal, Naval Ocean Systems Center-Hawaii Laboratory, Box 997, Kailua, HI 96734.
}

mations of alphabetic characters often perceptually created stimulus components that were not physically present, but were only suggested by the arrangement of the other parts of the stimulus. The ability to perceive wholeness in a world of naturally masked stimuli and noisy or incomplete views of objects is obviously a very valuable skill.

The question of how we fill in or complete partial or sampled stimuli is also of considerable interest to students of computational vision. The task of computational vision, as idealized by Marr (1982), is to provide a formal model of the transformation steps that "must" occur between the input (the physical stimulus) and the output (the perceptual experience itself). Marr argued that identification of these transformational steps might be an appropriate goal of vision researchers, even though the exact mechanisms or neural algorithms that produced these transformations might remain impenetrable.

Examples of perceptual reconstruction abound in the literature of both computational and conventional vision research and in the everyday experience of human observers. We take note particularly of the subjective surface phenomena (Bachmann, 1978, 1980; Kanizsa, 1974; Petry \& Meyer, 1986) and of the elegant studies of the reconstruction of geometrical form (1) from two or more sequential views of a moving object (Braunstein, 1988; Lappin, Doner, \& Kotas, 1980; Lappin \& Fuqua, 1983; Ullman, 1979), (2) from shading and lighting (Horn, 1975), (3) from the disparities among sparsely sampled random dot stereograms (Grimson, 1981; Julesz, 1960, 1971), and (4) from two-dimensional projections of curvature (Stevens, 1986). 
The specific challenge confronted in the present work was to determine how observers transformed sparsely sampled (with dots), three-dimensional, geometrical surfaces into a perceptual appreciation of their overall global form. Phenomenologically, three-dimensional subjective surfaces, which are akin to the two-dimensional subjective surfaces brought to our attention by Kanizsa (1974), appear when the dot densities on that sampled surface are relatively high. In similar experiments, in which much higher sampling dot densities were used (Uttal, 1987), observers reported that the stereoscopically plotted, pointlike dots appeared to be supported on a dark velvet-like background shaped like the continuous surface from which the dots were sampled. This phenomenon mirrors some of the properties of the two-dimensional Kanizsa figures.

Those high-density versions of sampled surfaces could be detected, discriminated, and recognized with a high degree of visual efficiency even when they were degraded by the presence of large numbers of extraneous "masking" dots randomly positioned in the three-dimensional viewing space. Accordingly, we turned to the question of what happens to the ability to reconstruct the original geometrical surfaces with a different type of stimulus degradation, that is, when the number of dots is relatively small. Specifically, we wanted to determine the smallest number of dots necessary to perceptually define quadric surfaces (generated from a second-order polynomial) and cubic surfaces (generated from a third-order polynomial).

The measurement of perceptual reconstruction poses a serious methodological challenge to the experimenter. Although we would have preferred to determine exactly what was actually perceived by each observer when presented with a sparsely sampled stimulus, obviously such an inspection of an intrapersonal percept was an impossible goal. Therefore, as a measure of perceptual reconstruction, we used the ability of the observer to "recognize" the specific form from which the dots were sampled, that is, to name the surface. Our metric of reconstruction, therefore, was necessarily indirect and, like all behavioral estimates of any kind of cognitive process, only suggestive of what the observer actually perceives.

In short, we shall show that the performance of the human observer is actually superior to that of a simple model of reconstruction-a least squares, polynomial, surfacefitting expression. This simple expression constitutes an initial theoretical approximation to geometrical image reconstruction by the human observer, but it is obviously incomplete, and it might be totally incongruous with what the human visual information system actually does.

A similar outcome, in which the human observer exceeds the performance of an "ideal" observer, has been reported by Braunstein (1988) with regard to the reconstruction of a dotted form. However, in that study the salient cue was invariant under translation and rotation rather than stereoscopic disparity. The superiority of the observers' performance over that of the model in both of ther cases must be interpreted to mean that the observer takes advantage of cues that are not incorporated into either of the models. An additional part of our task, there- fore, was to identify these additional cues or constraints that allowed the observer to recognize three-dimensional geometrical forms when they contained what initially seems to be an impossibly small number of samp!e dots.

Three experiments, differing in slight detail to allow us to compare performance between experimental conditions, were carried out. Experiment 1 differed from Experiments 2 and 3 in the size and shape of the stimulus forms. Experiment 3 differed from Experiments 1 and 2 in the orientation of the stimulus forms: the stimulus forms in Experiment 3 were rotated to two orientations other than the standard vertical one used in the other two experiments.

We also sought to determine whether there was any differential sensitivity to the specific three-dimensional forms of the stereoscopically generated stimuli. Although some such differential effects did emerge, the raw effects of form per se were not stable from experiment to experiment. In spite of a high level of intraexperimental stability and reliability, when plotted simply as a function of the stimulus form, the observed effects turned out to be idiosyncratic; they differed within the three present experiments, and they also differed from the results reported in the earlier, closely related experiments (Uttal, 1987). This result reinforced our feeling that recognition experiments of this nature produce results that are extremely sensitive to very modest changes in procedure. Gross qualitative changes in performance could often be induced by what a priori seemed to be trivial changes in experimental design. On the other hand, stable form effects were discovered when the experiments were analyzed with confusion matrices-an alternative means of studying the data.

\section{METHOD}

\section{Observers}

Three stereoscopically competent observers were used in this study. None had any uncorrected visual aberrations. All had considerable experience in the procedure.

\section{Apparatus}

The study was carried out using a computer-controlled system that generated the displays, collected the responses, and automatically analyzed the outcomes. The stimuli consisted of sparsely sampled $(2<n<20$ ) random-dot stereograms of planar, quadric, and cubic three-dimensional, single-valued surfaces similar to those originally used by Julesz (1960) in his studies of cyclopean vision. The stimulus surfaces were generated in the form of dichoptic stereograms on the face of a Hewlett-Packard 1311-B point-plotting oscilloscope with a fast decay ( $\mathrm{P}-24)$ phosphor. The manufacturer assured us in the technical manual that this signal trace would diminish in luminance down to .1 of $1 \%$ of its original value after a few microseconds. The specified duration of the stimulus presentation in this study was 1 sec. Previous experiments, however, had shown no significant effects of duration per se even when the stimulus duration was prolonged to 2 or $3 \mathrm{sec}$. Each dot was displayed at a luminosity well above threshold, although luminosity is also known not to influence performance in this range.

The observers viewed the screen from a distance of $71 \mathrm{~cm}$. Each of the dichoptic images subtended a visual angle of $5.25^{\circ} \times 5.25^{\circ}$ on the face of the oscilloscope for Experiment 1 and $4.25^{\circ} \times 4.25^{\circ}$ 
for Experiments 2 and 3. Rotary prisms were placed in front of each eye and individually adjusted by each observer to allow comfortable ocular convergence. The third (depth) dimension of the cube was defined by the dichoptic disparity of the left-eye and right-eye images as specified in the computer program. Disparity was initially adjusted by the experimenter so that the volume enclosed was judged to be approximately cubical. The disparity limits, thus arbitrarily defined, were subsequently determined to range from $14^{\prime}$ of crossed disparity to 14' of uncrossed disparity, with fixation (zero disparity) always placed at the center of the viewing cube. Figure 1 shows the appearance of one of the sparsely sampled dichoptic stimuli. If viewed through a stereoscope, this stereogram will appear as a paraboloid of rotation convex toward the observer.

The images generated on the face of the oscilloscope were created from algorithms and stored prototypes within a Cromemco S-3 microcomputer. The digital representations of the three-dimensional forms in the computer memory were then converted by means of digital-to-analog converters into three voltage-varying signals representing the $x, y$, and $z$ coordinates of a Cartesian three-space. The voltages were then fed into a special-purpose analog computer constructed from Optical Electronics components, which converted the voltages representing the triplet set of numbers encoding the three-dimensional coordinates of each dot into two voltages representing pairs of numbers encoding the two-dimensional coordinates of a disparate pair of dots-the left- and right-eye haploscopic images, respectively. The analog computer allowed this conversion to be carried out in real time without burdening the digital computer with extensive and repetitive trigonometric calculations.

\section{Stimuli}

The stimulus forms used throughout this study consisted of seven stereoscopically generated, nonplanar surfaces and one plane. Diagrams of these eight forms are shown in Figure 2. However, these diagrams are not true depictions on two counts: (1) They show the general shapes but not the exact depth dimensions, which varied from experiment to experiment. (2) There are no grid lines or outline cubes in the actual stimulus presentations; they appear simply as a group of punctate dots distributed randomly in the viewing space. The lines are presented here only to aid in visualizing the shape of the surfaces with the two-dimensional projections of Figure 2.

The stimuli were presented without any random masking dots (as was done in our previous research). In the present study, the stimulus degradation necessary to challenge the observer's visual system was implemented by simply reducing the number of dots

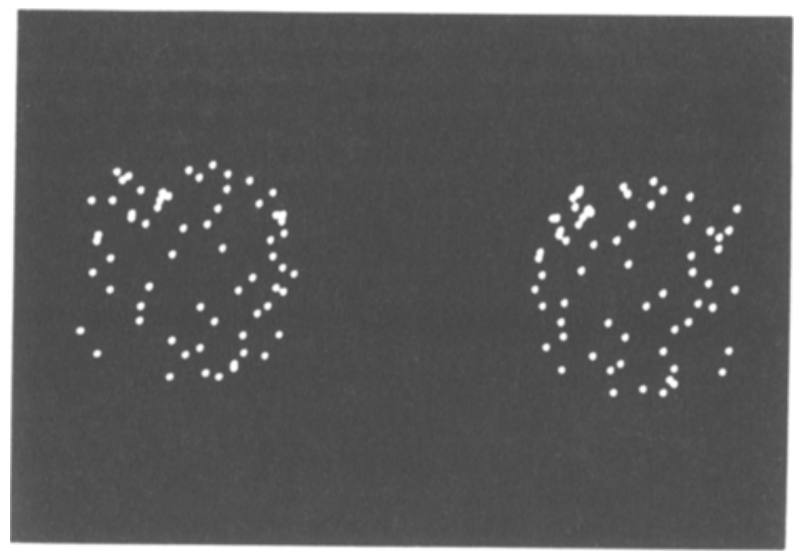

Figure 1. An example of the stimulus forms used in this experiment. If viewed through a stereoscope, this random-dot stereogram will appear as a paraboloid of rotation convex toward the observer.
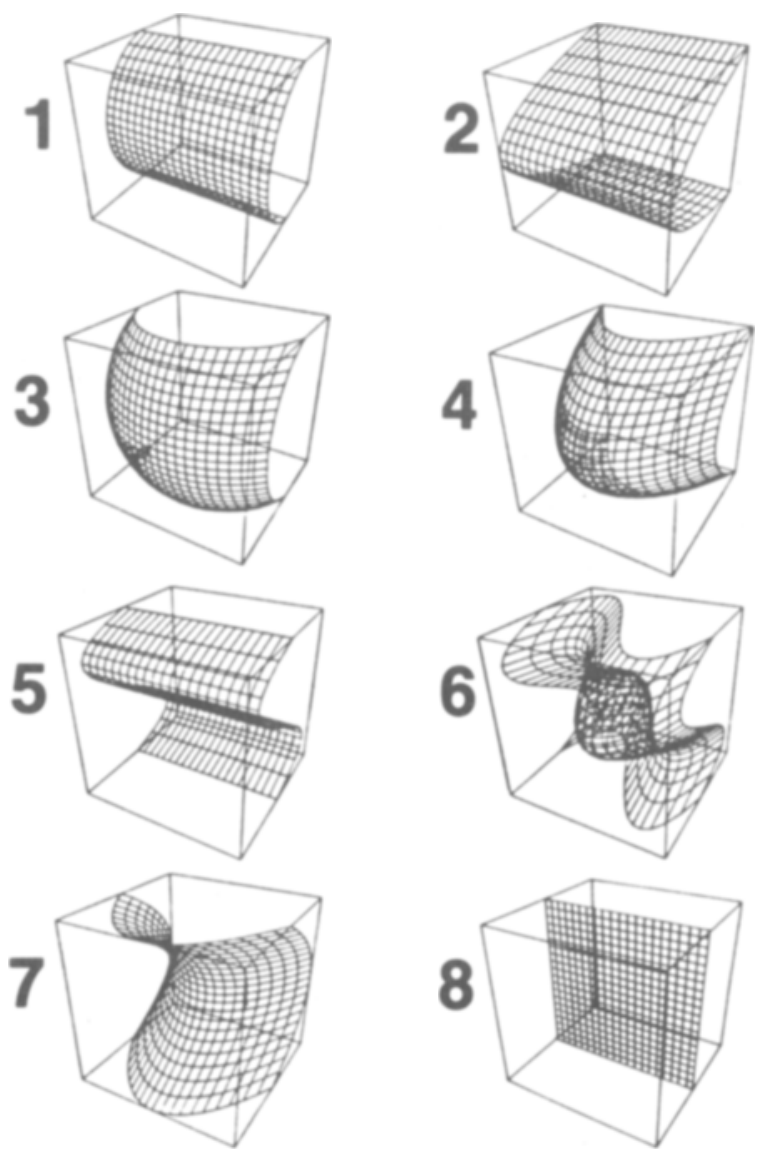

Figure 2. Diagrammatic sketches of the eight stimulus forms used in this study. The actual stimuli appeared as in Figure 1: The outlines and grid lines are presented here only to clarify the shape of these two-dimensional projections of three-dimensional images.

sampling the defined three-dimensional surfaces. All of the surfaces used as stimulus forms were created by the application of a polynomial equation (Equation 1 ) to a set of randomly selected $x, y$ coordinate pairs corresponding to positions on a plane. In Experiment 1, the plane, hyperbolic paraboloid (saddle), one-dimensional cubic (cubic1D), two-dimensional cubic (cubic2D), parabolic arch, and cylinder were constructed from the same set of random $x, y$ pairs placed within a square prototype. The paraboloid of rotation and the hemisphere, on the other hand, were constructed from a different set of random $x, y$ pairs placed within a decagonal prototype. Figure 3 presents these planar prototypes. In Experiments 2 and 3 , all of the stimuli were generated from the same random dots placed in a square prototype. This was done to determine whether subjective outlines defined by the array of dots had any influence on our results, and to provide a replication with which to evaluate interexperimental variability. As we shall see, the outlines seem to have had a negligible effect on performance, and interexperimental variability is quite small.

The polynomial expression that "stretched" the planar prototype surface into the seven three-dimensional nonplanar surfaces is

$$
\begin{aligned}
z= & \mathrm{G}\left(\mathrm{A} x^{5}+\mathrm{B} x^{4}+\mathrm{C} x^{3}+\mathrm{D} x^{2}+\mathrm{E} x+\mathrm{R} y^{5}+\mathrm{S} y^{4}\right. \\
& \left.+\mathrm{T} y^{3}+\mathrm{U} y^{2}+\mathrm{V} y+\mathrm{W}\right)^{\mathrm{F}}+\mathrm{H} .
\end{aligned}
$$

By arbitrarily selecting the coefficients (A, B, C, D, E, F, G, H, $R, S, T, U, V$, and $W$ ) in this equation (including, of course, the option to set each to zero), it is possible to produce a wide variety 

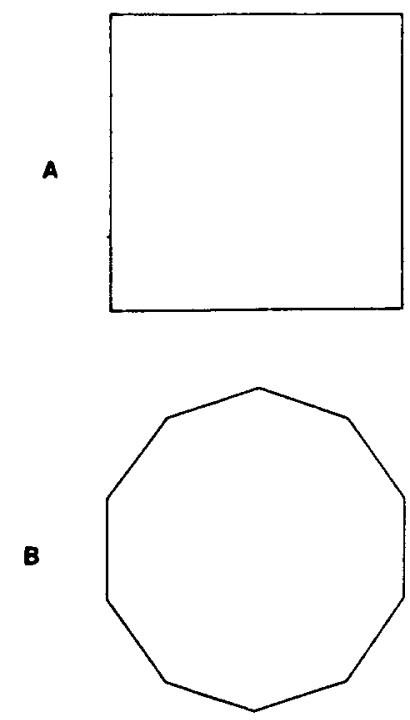

Figure 3. The outlines of the two planar prototypes used in Experiment 1. The planar prototype used in Experiments 2 and 3 for all stimulus forms was a square, but $1^{\circ}$ of visual angle smaller than square A.

of surfaces. The polynomial expressions for the eight stimulus forms used as stimuli in this study are presented in Table 1 . The numerical values indicated in this table are not generally meaningful; they are specific to our computer programs and the nature of the digitalto-analog conversion process used in our computer system. The geometrical surfaces used as stimuli were all aligned in a frontoparallel plane with the main axis of convexity apparently protruding toward the observer by appropriate selection of the disparities.

In Experiment 3, the same stimulus set was used as in Experiment 2 with only one important change: The eight stimuli were presented at random in three different orientations $-0^{\circ}$ (vertical) and tilted either clockwise or counterclockwise $45^{\circ}$. This was done to determine if orientation played a role in recognition of the stimulus forms, and to serve as a control for local and fixed-position feature cues that might have been inadvertently introduced into our stimuli. The minimal effects of form support the argument that this did not happen, and that our observers' performance was, indeed, driven by the global shape of the stimulus forms.
In Experiment 1, there were eight stimulus forms presented at 9 different dot numerosities; in Experiment 2, the eight forms were presented at 11 dot numerosities; and in Experiment 3, the 24 stimuli ( 8 forms $\times 3$ orientations) were presented at 10 dot numerosities. Therefore, in each of these three experiments, the major independent variables were the number of sample dots, the size and shape of the prototype plane, the shapes of the stimulus forms, and, in one of the experiments, the orientation of the stimulus form.

The particular dots that were selected for presentation in each trial varied randomly from among a larger, randomly positioned sample of dots on the original prototype form. In Experiment 1, 100 dots were distributed about the square prototype and 78 on the decagonal one. In Experiments 2 and 3, 64 dots were distributed about the smaller square planar prototype that was used to generate all of the stimulus forms. On each trial, a sample of a few dots was randomly selected from among these available dots. Because of the relatively large number from which the sample came (64, 78 , or 100 ), it is extremely improbable that the individual absolute location of a single dot, or even a particular configuration of a few dots, could become associated with any particular form.

Any potential absolute location cue was further controlled by the fact that all of the stimulus forms used in each experiment were sampled from the same set of random dots in their respective twodimensional prototypes. That is, with the exception of the hemisphere and paraboloid of rotation in Experiment 1, within each experiment the $x, y$ coordinates of all stimulus forms were the same. Therefore, without binocular disparity as a cue, this task could not be carried out. We believed, rather, that in any given trial it would be the relative arrangement of the dots in the three-dimensional, stereoscopic space that would be the critical cue used by the observers in solving the perceptual problem posed to them by this experimental design. Our task was to determine which particular attributes of arrangement would allow our observers to reconstruct and recognize these sparsely sampled stimulus forms.

\section{Psychophysical Procedure}

This experiment was carried out in a recognition mode. That is, the observers were asked to orally enunciate the name of one of the eight stimulus forms-a set of verbal responses that had been learned in preliminary training sessions with high-dot-numerosity versions of the stimulus forms. An Interstate Electronics Corporation VR200 voice-recognition terminal was used to acquire responses. Because this device required "training" to respond to each speaker's voice, a preliminary procedure lasting approximately 3 min occurred at the beginning of each experimental session. Because of the moderate level of recognition accuracy provided by

Table 1

The Formulas Used to Stretch the Seven Nonplanar Stimulus Forms from the Prototype Surface

\begin{tabular}{ll}
\hline \multicolumn{1}{c}{ Stimulus Form } & \\
\hline Cylinder & $z=2535-\sqrt{948676-y^{2}}$ \\
Arch & $z=1099+.002 y^{2}$ \\
Hemisphere & $z=3221-\sqrt{1890625-x^{2}-y^{2}}$ \\
Paraboloid of Rotation & $z=1085+.002\left(x^{2}+y^{2}\right)$ \\
One-Dimensional Cubic & $z=2048+.0025\left(.001 y^{3}-948.676 y\right)$ \\
Two-Dimensional Cubic & $z=2048+.001\left(.001 x^{3}-948.676 x+.001 y^{3}-948.676 y\right)$ \\
Saddle & $z=2048-.001\left(x^{2}-y^{2}\right)$ \\
Plane & $z=2048$ \\
\hline
\end{tabular}


the VR200, it was deemed necessary to also have the computer respond through a VOTRAX voice-generation device with the utterance that had been acquired. The observer then accepted or rejected the computer's decision by pressing one of the two handheld pushbuttons. Thus, the intertrial timing of the experiment was controlled by the pace of the observer's responses.

In this recognition-type experiment, a single trial began with a 1-sec presentation of a dichoptic pair of fixation dots centrally placed in the viewing space. This pair of dots was used by the observers to define the zero-disparity fixation point, and thus helped to establish steady fixation, dot-pair correspondences, and good stereoscopic fusion. ${ }^{1}$

The pair of fixation dots was followed by a single stimulus form, which also appeared for $1 \mathrm{sec}$, and then by a briefly flashed question mark. The question mark helped the observers to synchronize their utterances with the acoustic "open window" during which the voice-recognition system was able to accept and process the response. Figure 4 shows the sequence of events in a single experimental trial. During each daily experimental session, the order of the stimulus forms was selected randomly, as was the number of dots on those days in which the dot numerosity varied.

Experiment 1 was carried out over 10 days, with dot-numerosity orders on Days 6-10 being replications of those on Days 5-1, respectively (thus pairing the 1 st and 10th days, the 2 nd and 9 th days, etc.). During the first 5 days, the stimuli were presented in overlapping but generally descending order of dot numerosity. On the first day, the stimuli consisted of 20,16 , or 10 dots. On the second, 10,8 , or 6 ; on the third, 8,6 , or 4 ; on the fourth, 6,4 , or 2 ; and on the fifth, 5 or 3 . The sequence was then repeated in reverse order. (See Figure 9.)

In Experiment 2, the general procedure was the same except that the experiment was run on 14 days with the sequence of dot numerosities varying from day to day in the following order: 20 , 16 , or $10 ; 10,8$, or $6 ; 9,7$, or $5 ; 8,6$, or $4 ; 7,5$, or $3 ; 6,4$, or 2 ; and finally, 5 or 3 , for the first 7 days. This series was then also repeated in reverse order. (See Figure 10.)

In Experiment 3, the sequence was different. Since the eight stimuli were shown in three different orientations, only one dot numerosity could be used each day. On 10 successive days, 16, $10,9,8,7,6,5,4,3$, and 2 dots were used, respectively; the sequence was then also run in the reverse order. (See Figure 11.)

\section{RESULTS}

Rather than discussing the results of each experiment separately, we present the corresponding parts of the three experiments together. Data are organized into three major categories: (1) the effect of the raw stimulus form on recognition, (2) the effect of the number of dots on recognition, and (3) the confusion matrices. The data were analyzed by a system of special-purpose statistical programs. Initially, this was done by a program that computed confusion matrices for all combinations of the eight stimuli and also produced graphs of the percentage of correct recognition as a function of the other independent variables. Subsequently, other analysis programs were used to pool the data from individual observers and sessions as indicated in the next section.

\section{The Effect of Stimulus Form}

Figure 5 shows the results plotted as a function of the eight stimulus forms for Experiments 1, 2, and 3, respectively. Each of the three curves in this figure plots the percent correct scores as a function of the shape of the stimulus form in the sequence (1) cylinder, (2) parabolic arch, (3) hemisphere, (4) paraboloid of rotation, (5) onedimensional cubic, (6) two-dimensional cubic, (7) hyperbolic paraboloid, and (8) plane. On the curve that presents the results of Experiment 1, each data point summarizes the outcome of approximately 750 trials using sparse samples of the 78 or 100 possible dot positions. Each data point on the curves detailing the results of Experiments 2 and 3 represents the pooled results of approximately 1,000 trials in which that particular form was chosen at random from the eight possibilities and in which the particular set of sparsely sampled dots on each form was randomly sclected from the 64 possible dot positions. Since the scores of individual observers and conditions have been pooled into cumulative percent correct scores, statistical tests of significance are not appropriate. Because of the relatively large numbers of trials so pooled, statistical tests are not necessary.

The curve in Figure 5 that depicts the results of Experiment 1 shows a marked elevation of performance for both the planar surface and the paraboloid of rotation. The two are a curious pair because the paraboloid is among forms with the greatest possible maximum difference in depth between the farthest and the closest sample points, while the planar surface contains the least. Although these data diverge from other, comparable data discussed below, there is little variation of the shape of this curve

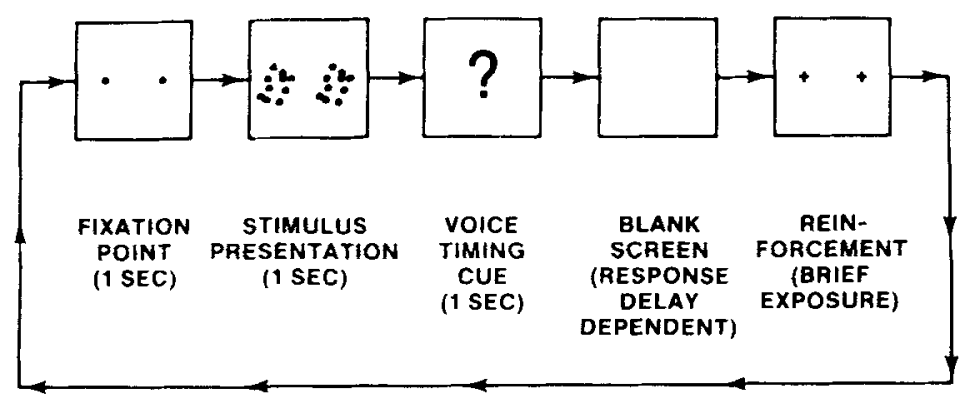

Figure 4. The sequence of events in a single experimental trial. 


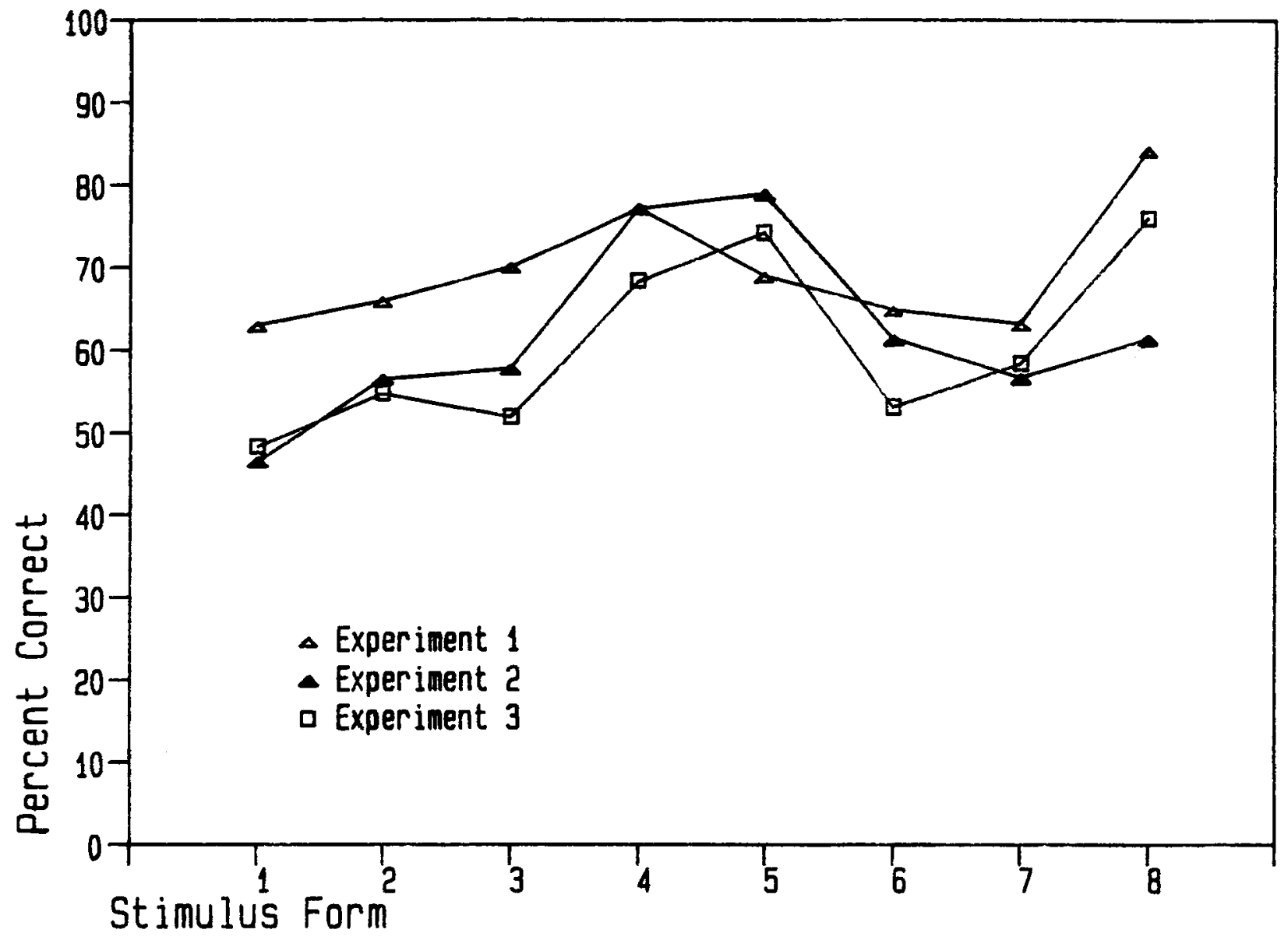

Figure 5. The results of Experiments 1,2, and 3 plotted as a function of the stimulus form (pooled results). On this and all subsequent figures plotted as a function of this same variable, $1=$ cylinder; $2=$ parabolic arch; $3=$ hemisphere; $4=$ paraboloid of rotation; 5 $=$ cubic in one dimension; $6=$ cubic in two dimensions; $7=$ hyperbolic paraboloid; $8=$ surface.

among the observers used in this experiment, as shown in Figure 6. Each point on this figure represents the pooled result of approximately 250 trials.

The curve in Figure 5 that depicts the results of Experiment 2 as a function of stimulus form shows a somewhat different pattern of performance. In this case the two stimulus forms that are reconstructed and recognized best are the paraboloid of rotation and the one-dimensional cubic. Little difference in performance exists for any of the other stimulus forms. In spite of the fact that, in Figure 5, the shape of the curve for Experiment 2 differs from that of Experiment 1, there is considerable stability among the daily sessions, which varied in the number of sampled dots. Figure 7 shows the Experiment 2 data segregated on the basis of the daily runs; obviously the same shape of the form curve is replicated from day to day. Each point on this figure represents the pooled result of over 330 trials.

The third curve in Figure 5 shows the results for Experiment 3 . In this case the paraboloid of rotation and the one-dimensional cubic are joined by the plane as the most recognizable stimulus forms. Indeed, the plane surface is now the most recognizable. As shown in Figure 8, when these data are analyzed in terms of the orientation of the form, these results are also stable and reliable within the context of a single experiment. Each point on this figure represents the pooled result of over 330 trials.

Thus, as indicated in Figures 6, 7, and 8, when analyzed by observer, by run, or by orientation, the interexperimental differences we have obtained do not appear to be reflected in equivalent intraexperimental variability. We believe that these idiosyncratic interexperimental differences result primarily from shifts in strategy due to unknown aspects of our experimental design. When the data are analyzed as a function of the raw stimulus form, the differing results of the three experiments therefore preclude any exact conclusion with regard to the general nature of a putative stimulus-form effect.

Furthermore, the stimulus-form effects shown in these three figures also differ from those obtained in an earlier study (Uttal, 1987) in which essentially the same dotted forms were used, but with much higher dot numerosities, in comparable detection, discrimination, and recognition experiments. 


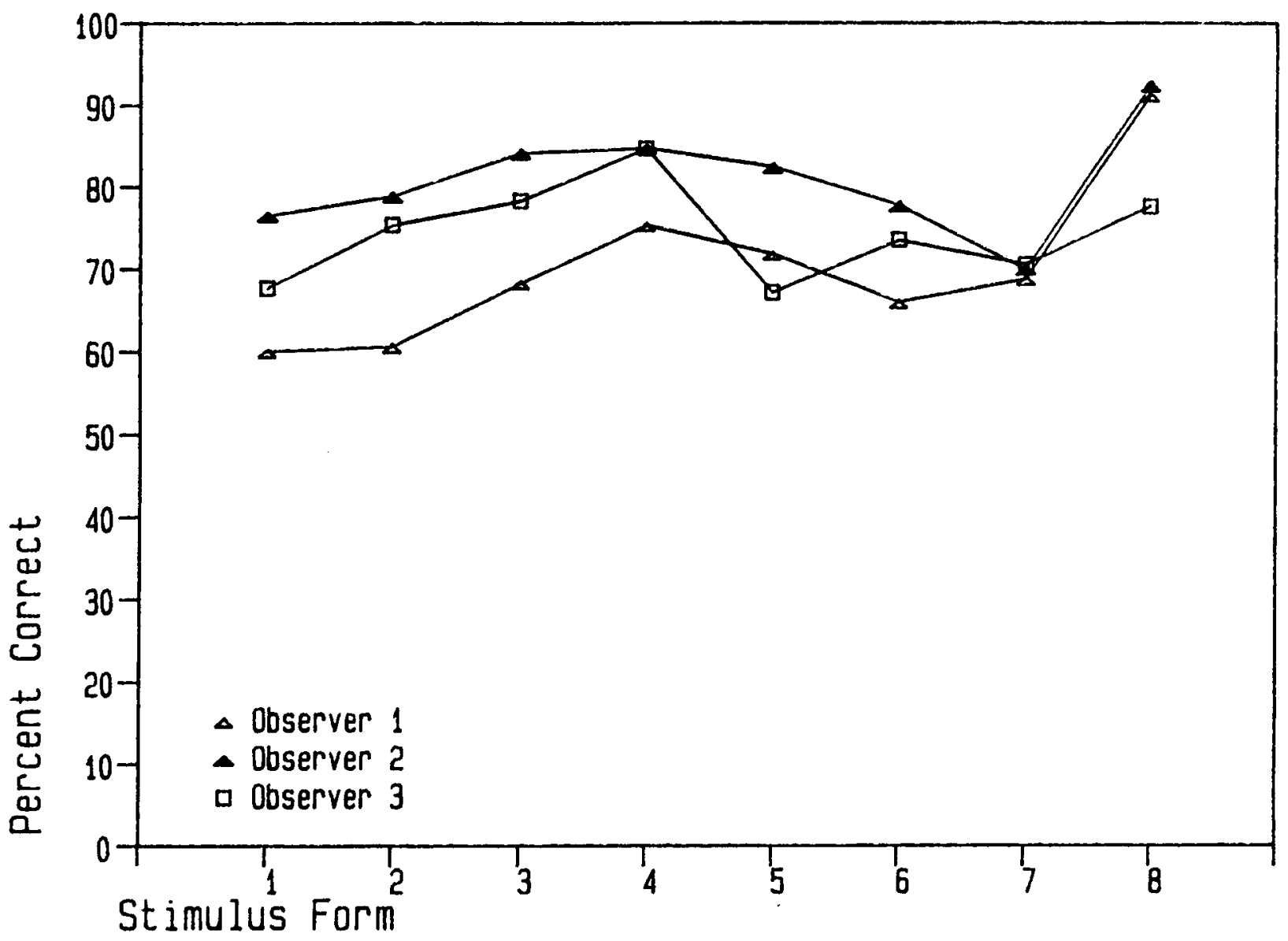

Figure 6. The results of Experiment 1 factored out on the basis of the 3 observers.

\section{The Effect of Dot Numerosity}

Figures 9, 10, and 11 summarize the results of Experiments 1, 2, and 3, respectively, when they are analyzed in terms of dot numerosity. Although the set of results discussed in the previous section reflected substantial interexperimental instabilities in the functional curves describing the data, analysis in terms of dot numerosity displays a high degree of stability in our observers' performance. The variability in the shape and magnitude of these three data sets is, obviously, small.

Figure 12 displays the results from Experiment 3 in another way-factored out according to the orientation of the surface. In this case, there is a noticeable difference between the two nonvertical orientations and the vertical orientation when the dot numerosity is relatively high $(>6)$. However, this difference disappears when the number of dots is small $(\leq 6)$.

The most striking finding of this analysis in terms of dot numerosity is the remarkably small number of dots required by the observer to identify which of the eight surfaces was represented by the dots. It should be noted that even with the smallest number of dots utilized (2), performance levels were above chance, and with only 4 sample dots performance levels of $50 \%$ were obtained. Also note that the curve essentially asymptotes at virtually perfect performance with 8 or 9 dots.

\section{The Confusion Matrices}

As noted above, one of our initial disappointments in analyzing these data was that the curves relating performance and stimulus form (as shown in Figure 5) seemed to be so variable from experiment to experiment, in spite of the high degree of intraexperimental reliability. The stable effect of form that had originally been the target of this research program therefore turned out to be elusive at this level of analysis. No general rules could be formulated describing the relationship between the shape of our stimulus forms and the performance scores. On the other hand, we did eventually discover that the confusion matrices obtained in this type of recognition experiment do display more stable form effects than those obtained in the other kind of analysis. In earlier work with densely sampled stimuli masked with random noise dots (Uttal, 1987), the obtained matrices displayed a specific pattern of confusions. The clusters of confused stimuli obtained in that earlier study were (1) the cylinder, the 


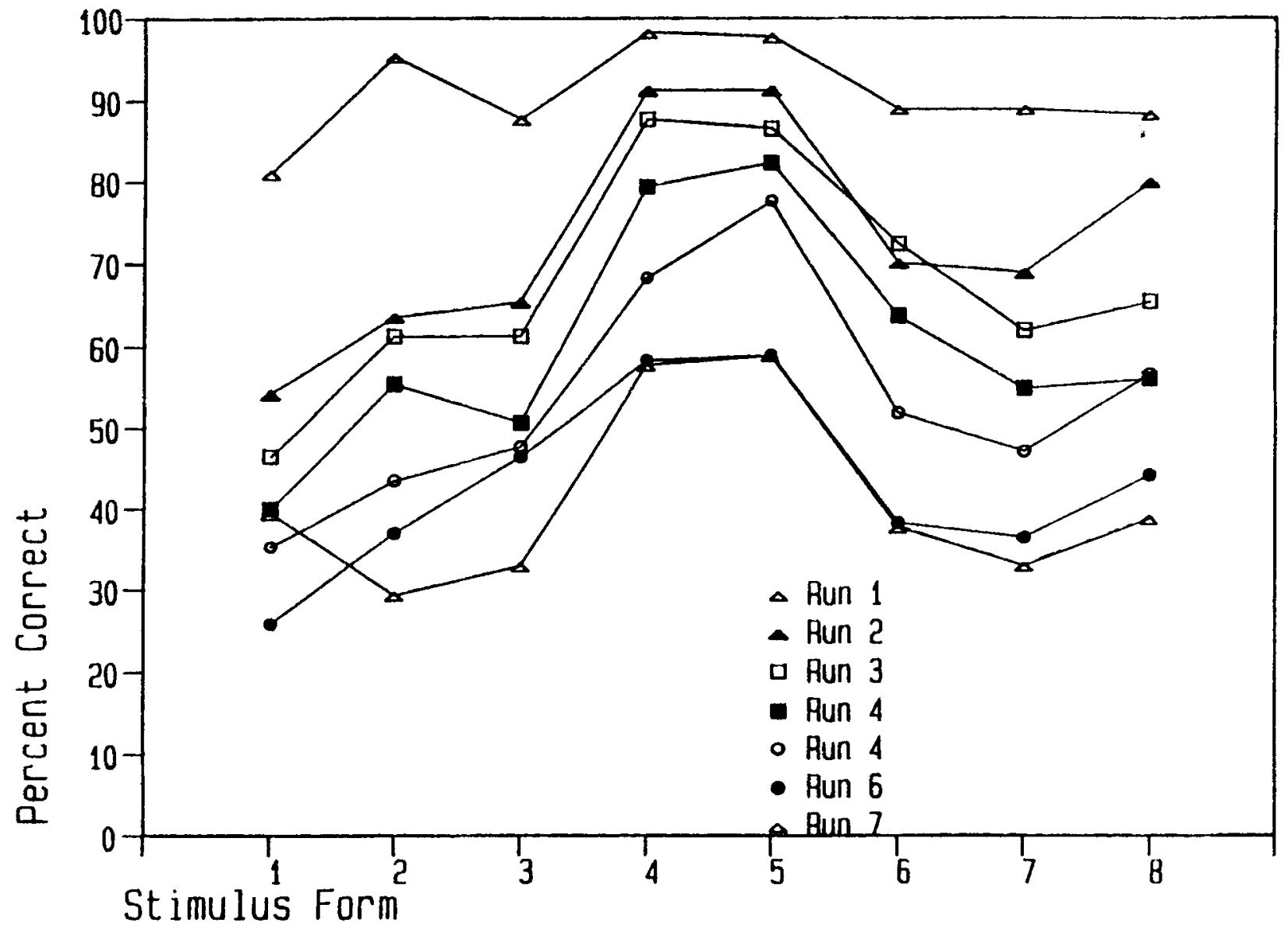

Figure 7. The results of Experiment 2 factored out on the basis of the seven runs.

parabolic arch, and the saddle; (2) the hemisphere and the paraboloid of rotation; and (3) the one-dimensional cubic and the two-dimensional cubic. (The plane was confused with the two-dimensional cubic but not with the onedimensional cubic.)

In the present study we also obtained relatively stable confusion clusters. However, these clusters were quite different from those unearthed in the earlier work. In our opinion, this difference is truly task dependent, and results mainly from the procedure in the previous series of experiments, which required the observers to detect, discriminate, or recognize a relatively densely sampled stimulus form within a noisy environment. In the present study, the observer was required to reconstruct the stimulus forms from relatively sparse samples in a noise-free environment. Therefore, a different set of cues and strategies was used by the observers in the perceptual processing of this new task.

One of the predominant characteristics of the confusion matrices from previous experiments was a strong diagonal symmetry indicating that response biases were neither prevalent nor influential in dictating the nature of our results. Diagonal symmetry is also characteristic of the results of the present study. Diagonal symmetry of this kind can be interpreted to mean that the responses were, in fact, determined by the properties of the stimulus forms rather than by any predilection on the part of our observers to respond preferentially with one or more of the names of the eight stimulus forms. Because of the diagonal symmetry, we can statistically fold Tables 2,3 , and 4 over their diagonals to produce pooled versions of the two halves of the data. This has been done in Tables 2,3 , and 4 by simply averaging the two corresponding scores from diagonal cells. Of course, the elements along the diagonals of these matrices are not error scores, but are the same as the percent correct scores plotted in Figure 5.

A further clarification of the results was obtained by tabulating only scores greater than $5 \%$. We then combined the results of all three experiments, so reduced, into a single summary (Table 5) whenever two or three entries with scores greater than $5 \%$ were available to be averaged. That average was then entered into the corresponding cell of the summary table.

There are a number of differences between this summary table and the summary table from the earlier research (Uttal, 1987). The high degree of confusability of the hemisphere and the paraboloid of rotation observed in the earlier study no longer obtains; these cells are empty 


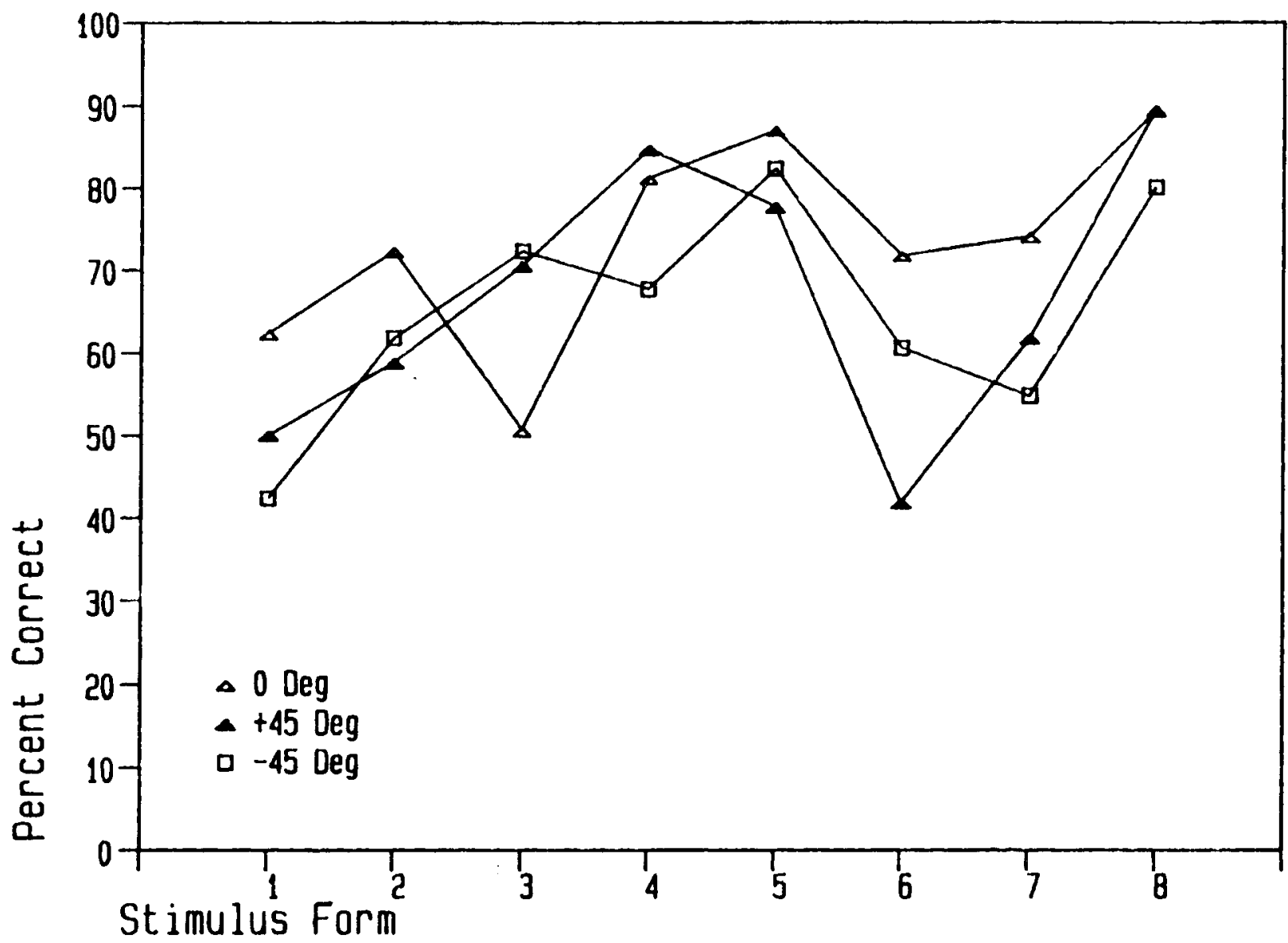

Figure 8. The results of Experiment 3 factored out on the basis of the orientation of the stimulus forms.

in these tables. The strong confusion cluster of the cylinder, the arch, and the saddle has been augmented by an even stronger confusion of the cylinder with the hemisphere and the plane. The arch, which shares a crosssection with the paraboloid of rotation, is now most strongly confused with the paraboloid, and also with the saddle. However, the paraboloid is confused only with the arch, but not with the forms with which the arch is confused. The one-dimensional and two-dimensional cubics are now strongly confused with each other in spite of the fact that they are very different in their respective cross-sections. The plane, not unexpectedly, is confused with the cylinder and the hemisphere-the next two shallowest stimulus forms.

This pattern of confusions will be considered in the discussion section.

\section{DISCUSSION}

\section{A Mathematical Anchor}

To anchor our psychophysical results, we need an answer to the question of how many sample dots are required by a mathematical algorithm to reconstruct the shape of the type of quadric and cubic surfaces that were used as stimuli in this study. We can then compare this answer with the answer to the psychophysical question of how many dots are required for the human observer to accomplish the same task. An initial mathematical answer is straightforward. If we observe that the quadric surfaces are represented in our stimulus set by the general equation

$$
z=\mathrm{G}\left(\mathrm{D} x^{2}+\mathrm{Ex}+\mathrm{U} y^{2}+\mathrm{V} y+\mathrm{W}\right)^{\mathrm{F}}+\mathrm{H},(2)
$$

and that we are asking only that the general class of surface (i.e., "Is it a hemisphere?" and not "Which specific hemisphere is it?"), then we may assume that there are only five unknowns ( D, E, U, V, and $F$ ) in this equation; $\mathrm{G}, \mathrm{W}$, and $\mathrm{H}$ may remain undefined. A minimum of 5 dots (i.e., five solutions to the equation, each of which is represented by a triplet number-the $x, y$, and $z$ coordinates of a point) would thus be required to designate a quadric surface. Similarly, if we assume that the cubics are represented by the equation

$$
\begin{aligned}
z= & \mathrm{G}\left(\mathrm{C} x^{3}+\mathrm{D} x^{2}+\mathrm{E} x+\mathrm{T} y^{3}\right. \\
& \left.+\mathrm{U} y^{2}+\mathrm{V} y+\mathrm{W}\right)^{\mathrm{F}}+\mathrm{H},
\end{aligned}
$$

then a minimum of 7 dots would be needed to reconstruct the form if only the class of the surface (and not the specific surface) must be determined. $\mathrm{G}, \mathrm{W}$, and $\mathrm{H}$ remain undefined in this case. 


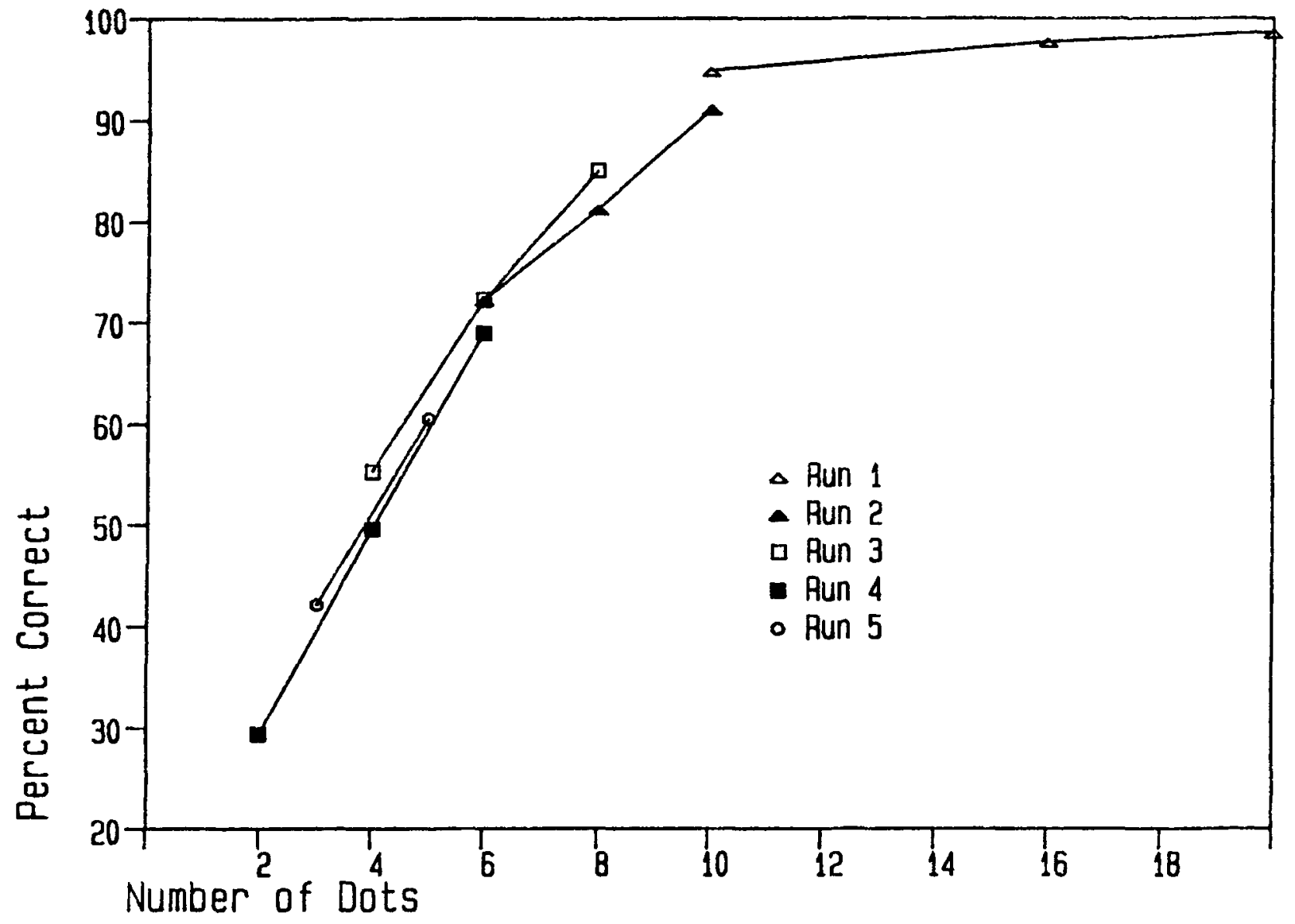

Figure 9. The results of Experiment 1 plotted as a function of the number of dots sampling the stimulus form.

The human observer, however, actually outperformed these reference anchors in the three experiments of this study. Performance was nonrandom, with fewer dots required for perceptual reconstruction (i.e., 4 dots allowed a $50 \%$ correct recognition) than even the relatively small number ( 5 and 7) suggested by Equations 2 and 3. Furthermore, the scores did not seem to systematically differ for the quadric and cubic stimulus forms. This additional discrepancy also suggests that the surface-fitting polynomial is not a good algorithm with which to model our observers' reconstructive abilities.

It should be made clear at the outset of this discussion, however, that there is no a priori reason that this model and the human observer should behave in the same way, and that this model need be as good as the human. It is very likely that the least-squares surface-fitting procedure modeled by these equations is a type of reconstruction process that is considerably different from that used by the observer to reconstruct or recognize the stimulus forms we utilized.

In spite of this dissociation between the predictions of this first-approximation model and the obtained human performance data, it is also true that any mathematical answer to the question of how many dots are necessary to reconstruct a form at least provides a reference against which to compare the psychophysical performance. If the mathematical model were complete, it would represent a truly ideal observer. The fact that our observer's performance differs from that of the surface-fitting model indicates that it is neither complete nor ideal.

In fact, the number of unique quadric and cubic surfaces is astronomically large. According to Hilbert and Cohn-Vossen (1952), there are infinity ${ }^{19}$ distinguishable cubic surfaces alone and infinity ${ }^{9}$ distinguishable quadric surfaces alone. Therefore, any arbitrary selection of either 9 or 19 points in space can, in principle, be fit with a unique quadric or cubic surface. In light of the very large number of possible surfaces, it is remarkable, as we have seen in Figures 9, 10, and 11, how few points are psychophysically necessary to designate that a sample of dots represents one of the stimulus surfaces shown in Figure 2.

The question we now face is, What additional information, constraints, or cues are used by the human observer in achieving the surprisingly high level of psychophysical performance measured in this study?

\section{Some Possible Perceptual Cues}

The scores shown in Figures 9, 10, and 11 (which are plotted as a function of the number of dots in the stimulus) establish that our observers' performance was very 


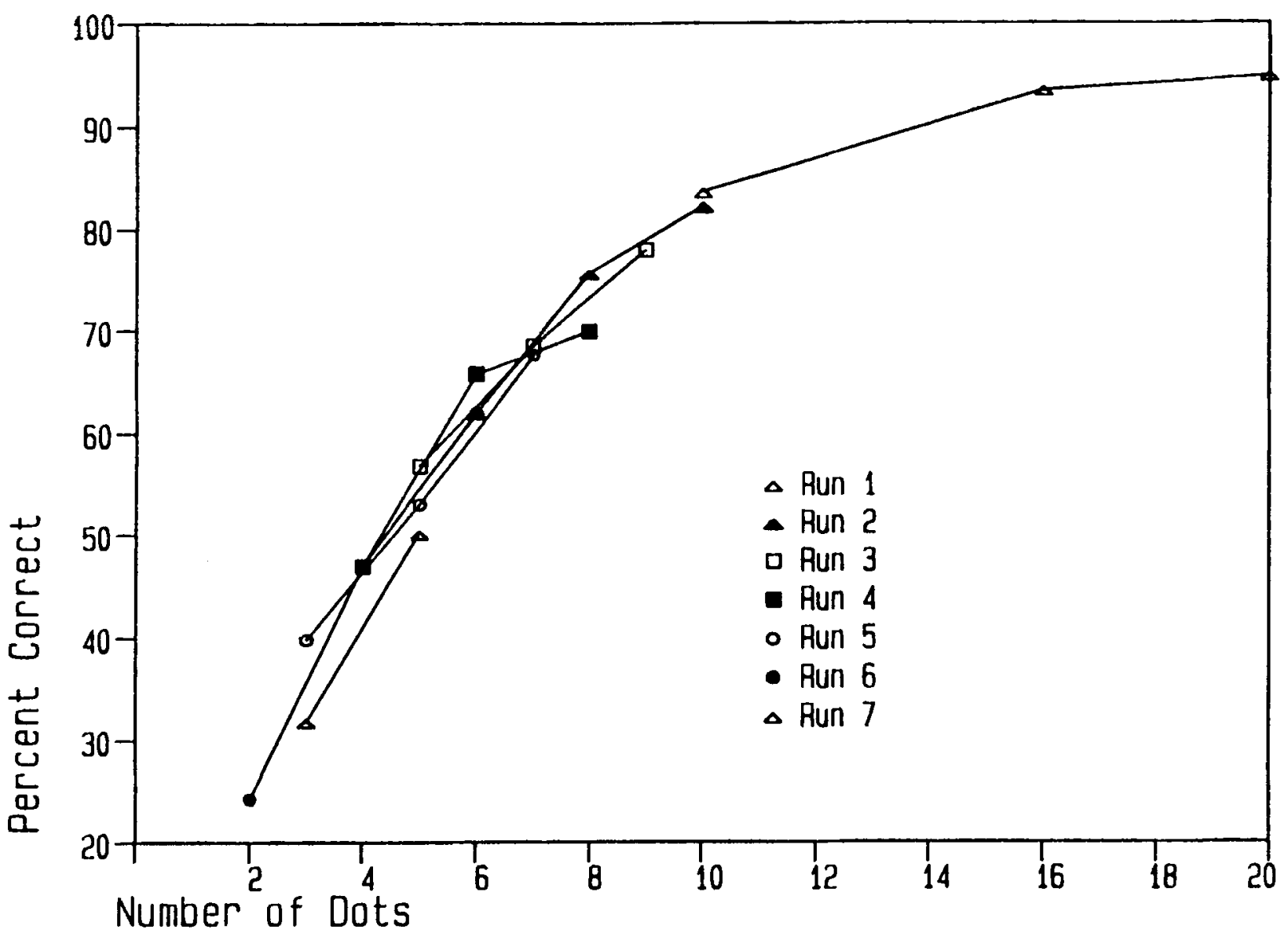

Figure 10. The results of Experiment 2 plotted as a function of the number of dots sampling the stimulus form.

good indeed. Fifty percent performance levels were consistently achieved with as few as 4 dots, and better-thanchance scores were obtained with even those stimuli that contained only 2 dots. (Random selections from among eight alternatives would predict $12.5 \%$ correct as the chance performance level.) However, as we saw, when the data were simply factored out as a function of the recognizability of the eight stimulus forms, little insight was obtained that could help us determine what information our observers were processing that allowed them to exceed the performance predicted by the curve-fitting Equations 2 and 3 . The set of most recognizable formsthe paraboloid of rotation, the one-dimensional cubic, and the plane, which were seen somewhat better than the other five forms-suggests nothing about the perceptual cues used by our observers. This particular cluster of three stimuli contains two of the four forms with the greatest depth and the one form with the least depth. There is also another form-the parabolic arch-that shares the same range of depths as those two; the one-dimensional cubic also has points in it that range broadly over the disparity dimension.

The confusion matrices, on the other hand, represent a rich lode of information concerning the nature of the additional cues for reconstruction that may or may not have been used by our observers. In the following paragraphs we will test various possibilities that may account for our observers' performance.

Curvature. First, we considered as one possible cue the degree of curvature exhibited by each of eight stimulus forms. Do stimulus forms with similar curvatures confuse with each other more than those with dissimilar curvatures? Four of our stimulus forms curve in two dimensions (the hemisphere, the paraboloid of rotation, the two-dimensional cubic, and the saddle), while the other four either have no curvature or curve in only a single dimension. Examination of the confusion matrix suggests that this attribute-curvature-is not likely to be a cue of any significance to our observers. Neither the paraboloid nor the hemisphere confuse with each other, and the paraboloid and the arch strongly confuse. Indeed, the strongest confusion observed in the matrix is between the cylinder and the hemisphere-stimuli with one and two dimensions of curvature, respectively.

Nor do such derived measures as the Gaussian curvature provide the criterion that would be required to account for these data. Gaussian curvature is defined as the product of the two orthogonal principle curvatures. The 


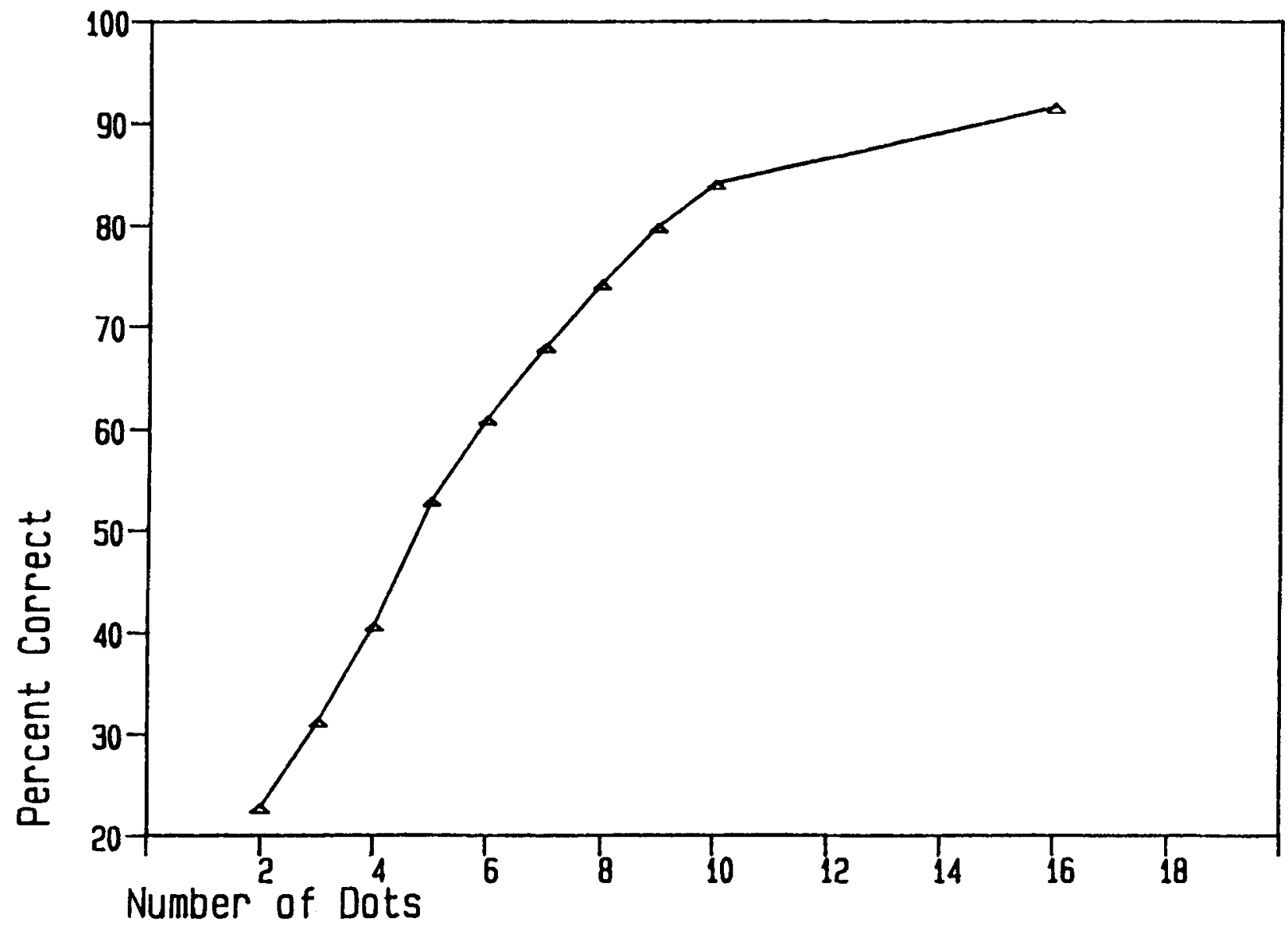

Figure 11. The results of Experiment 3 plotted as a function of the number of dots sampling the stimulus form.

Gaussian curvature, therefore, must be negative for a saddle (which has one negative and one positive axis of curvature) and positive for convex shapes. It must be zero for any surface that has at least one axis without curvature. Specifically, the signs of the Gaussian curvatures for the eight surfaces used in this study are the following:

$\begin{array}{ll}\text { Cylinder } & 0 \\ \text { Parabolic arch } & 0 \\ \text { Hemisphere } & \text { Positive } \\ \text { Paraboloid of rotation } & \text { Positive } \\ \text { One-dimensional cubic } & 0 \\ \text { Two-dimensional cubic } & \text { Somewhere positive } \\ & \text { and somewhere negative } \\ \text { Hyperbolic paraboloid } & \text { Negative } \\ \text { Plane } & 0\end{array}$

Since, for example, the confusions between the hemisphere and the cylinder (positive and 0 Gaussian curvatures), the arch and the paraboloid of rotation ( 0 and positive curvatures), and the saddle and the arch (negative and 0 Gaussian curvature) are among the strongest, similarity in the value of this metric is also a very poor predictor of human perceptual performance in the task posed by the conditions of this experiment.

Depth range. Other useful measures of the shapes of the eight stimulus forms can also be computed. Among these is the maximum depth range between the parts of each form that are closest to and farthest away from the observer. The range of possible depths in the computergenerated viewing space was 3,022 (in arbitrary units) at the back of the cube and 1,074 at the front, a maximum difference of 1,948 arbitrary units. The range of depth, for example, of the plane (stimulus form 8) is zero. The range of depth of the parabolic arch (stimulus form 2 ) in the stimulus set used in Experiment 1 is 1,874. Therefore, the value of the cell representing the difference in depth ranges between the paraboloid and the plane is 1,874 .

Even though they were generated by the same generating equations, the maximum and minimum depths of the stimuli used in Experiments 2 and 3 are different from those used in Experiment 1. This, however, can be attributed to the following: (1) The hemisphere and paraboloid of rotation were constructed from decagonal prototypes and thus do not extend as far back in their 


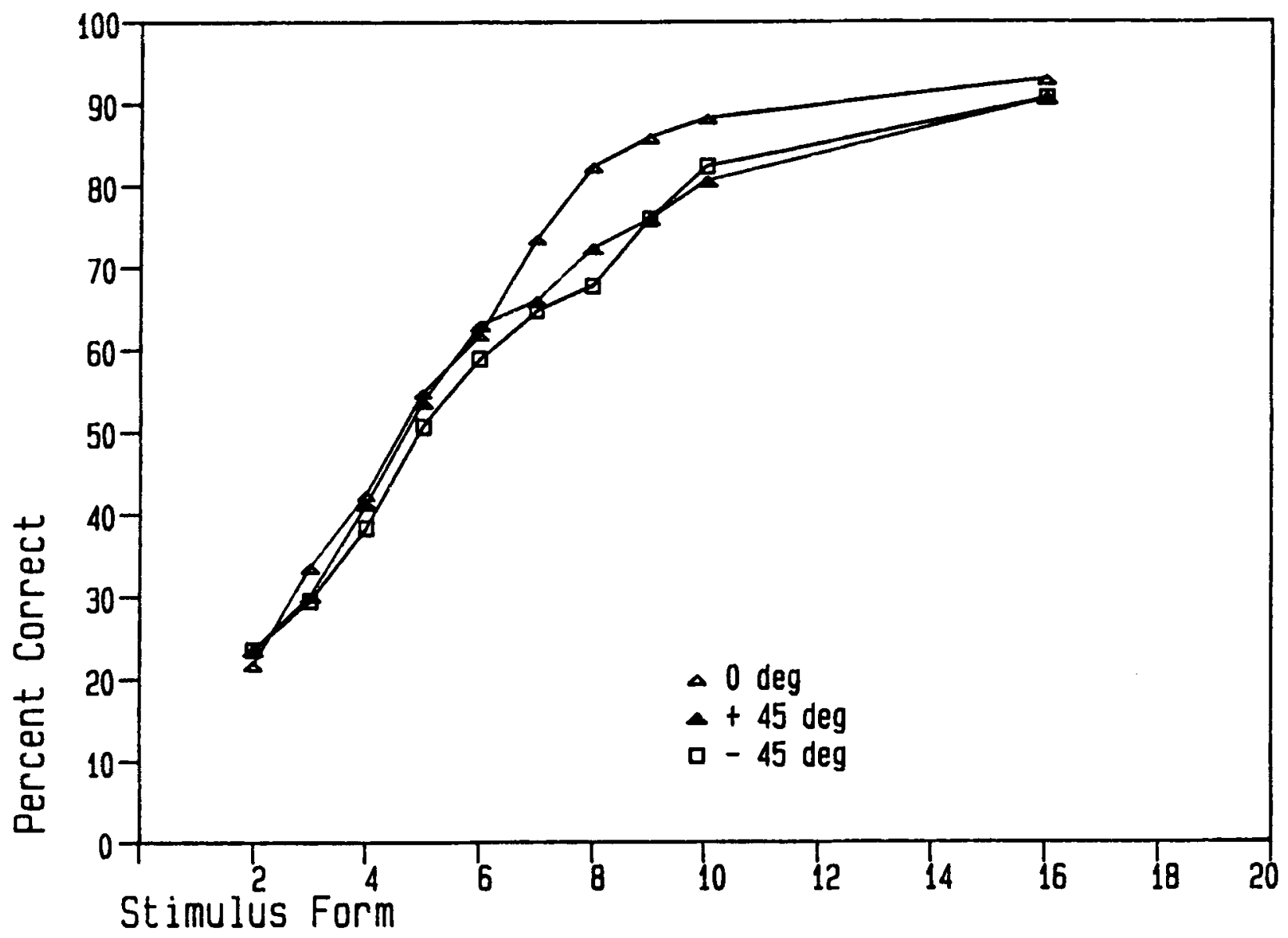

Figure 12. The results of Experiment 3 plotted as a function of the number of dots sampling the stimulus form, but factored out according to the orientation of the stimulus form.

corners as did the stimulus forms generated from square prototypes, and (2) the square prototypes used to generate the stimuli of Experiment 1 were $1^{\circ}$ larger than those used in the other two experiments. Although we had originally expected that such differences in prototypes might influence performance, this proved not to be the case. This lack of effect would help us later to determine the truly influential cues used by our observers in this task. The hypothesis to be tested in this case was that the greater the difference in depth ranges between any two stimulus forms, the less confusable they will be.

Enclosed volume. To measure the difference between the set of stimulus forms in a more global manner, we compared each stimulus form with all of the others by computationally determining the absolute value of the volume enclosed between each pair of surfaces when they were placed in the standard position in the viewing cube. The hypothesis to be tested in this case was that the larger the bounded volume, the less similar and, therefore, the less confusable are any two forms.

Slope. We then tabulated a third and somewhat more subtle attribute that may have helped our observers recognize the stimulus forms--the difference in typical slope between any two forms. An arbitrary measure of the typi- cal slope (SL) at any point on any form was defined as:

$$
\mathrm{SL}=\sqrt{\frac{\left(\left|\Delta z_{x 1}\right|+\left|\Delta z_{x 2}\right|\right)^{2}}{2}+\frac{\left(\left|\Delta z_{y 1}\right|+\left|\Delta z_{y 2}\right|\right)^{2}}{2}}
$$

where $\Delta z_{x 1}$ and $\Delta z_{x 2}$ are the differences in depth between the point in question and its two nearest neighbors in the $x$ direction, and $\Delta z_{y 1}$ and $\Delta z_{y 2}$ are the differences in depth of the point in question and its two nearest neighbors in the $y$ direction. This gives a single number representing the slope of each point on the surface. To compare two surfaces, we took the absolute difference of each pair of corresponding points on the two surfaces and summed them to give a single number $-\Delta S \mathrm{SL}-$ representing the difference in slopes of the surfaces. We determined the set of points used for the computation by placing a regular grid (equal $x$ and $y$ spacing) over the generating prototype plane. This procedure can be represented as

$$
\Delta S L=\left|S L_{1}-S L_{2}\right|,
$$

where $\mathrm{SL}_{1}$ and $\mathrm{SL}_{2}$ represent the slopes of the two corresponding points on the two surfaces. This is another global measure of the difference between any two sur- 
Table 2

The Folded Confusion Matrix for Experiment 1

\begin{tabular}{|c|c|c|c|c|c|c|c|c|}
\hline & Cyl. & Arch & Hemis. & Parabolo. & Cubic1D & Cubic2D & Saddle & Plane \\
\hline Cyl. & 63 & 5.5 & 12.5 & 2 & 0.5 & 3 & 4.5 & 6.5 \\
\hline Arch & & 67 & 1 & 9.5 & 7 & 3.5 & 8 & 0.5 \\
\hline Hemis. & & & 70 & 2.5 & 0.5 & 3 & 2.5 & 7.5 \\
\hline Parabolo. & & & & 77 & 3 & 3 & 4 & 0.5 \\
\hline Cubic1D & & & & & 70 & 9 & 4 & 1 \\
\hline Cubic2D & & & & & & 66 & 10.5 & 2 \\
\hline Saddle & & & & & & & 64 & 2 \\
\hline Plane & & & & & & & & 84 \\
\hline
\end{tabular}

faces, but one based on an approximation to the first derivative of their shapes rather than their shapes per se. The hypothesis to be tested in this case is: The larger the $\Delta S L$, the less confusable are any two forms.

Area. Finally, the surface areas of the eight stimulus forms-another possible attribute that may be used as a cue-were computed with a discrete approximation to a double integration. In this case, we sought to determine if larger differences in surface area produced lower confusions between any pairs of forms.

\section{Tests of Possible Perceptual Cues}

Depth range. First, we consider the impact of the differences in the ranges of depths. A typical stimulus form in this experiment consisted of a very small number of dots distributed in the three-dimensional, stereoscopically generated viewing space. The characteristics of this sample of dots varied depending on the surface from which they were taken. For example, the dots associated with the paraboloid of rotation, the parabolic arch, the one-dimensional cubic, and, to a slightly lesser degree, the hyperbolic paraboloid (the saddle) all varied over relatively wide ranges of disparity, and thus apparent depth, in our experimental trials. On the other hand, the group consisting of the remaining four stimuli-the cylinder, the hemisphere, the two-dimensional cubic, and, of course, the shallowest of all, the plane-and their disparities had a much smaller range of apparent depths.

If our observers were simply basing their judgments on the range of apparent depths of the sample dots, this strategy could account in part for the above-chance performance when only 2 dots were present on the sampled surface. This single piece of information-the relative range of the two pairs of sample dots-would, on the average, allow the observers to reduce their uncertainty from one of eight stimulus forms to one of four, an example of either the shallow or the deep groups of stimulus forms. Chance behavior would then be closer to the $25 \%$ performance level attained by our observers for the 2-dot condition in all three experiments than to the $12.5 \%$ level that was predicted without this constraint. In this case, we would expect the confusion clusters of stimulus forms to show minimal confusions between any stimulus pairs with widely varying depth-range differences, but to regularly confuse any that had only modest differences in their depth ranges. The confusion clusters do suggest that such a factor may be important and possibly could account for part of the measured performance.

To test this hypothesis, we calculated Pearson correlation coefficients among this maximum depth-difference

Table 3

The Folded Confusion Matrix for Experiment 2

\begin{tabular}{|c|c|c|c|c|c|c|c|c|}
\hline & Cyl. & Arch & Hemis. & Parabolo. & CubiclD & Cubic2D & Saddle & Plane \\
\hline Cyl. & 47 & 7 & 15.5 & 1.5 & 0.5 & 2.5 & 10 & 10.5 \\
\hline Arch & & 56 & 3.5 & 9 & 4 & 4.5 & 12.5 & 2.5 \\
\hline Hemis. & & & 58 & 2.5 & 0 & 3.5 & 4.5 & 15.5 \\
\hline Parabolo. & & & & 78 & 3.5 & 3.5 & 3.5 & 1 \\
\hline Cubic1D & & & & & 79 & 7.5 & 2.5 & 0.5 \\
\hline Cubic2D & & & & & & 61 & 7.5 & 6 \\
\hline Saddle & & & & & & & 56 & 5 \\
\hline Plane & & & & & & & & 61 \\
\hline
\end{tabular}

Note-Cyl. = cylinder, Hemis. $=$ hemisphere, Parabolo. = paraboloid of rotation. 
Table 4

The Folded Confusion Matrix for Experiment 3

\begin{tabular}{|c|c|c|c|c|c|c|c|c|}
\hline & Cyl. & Arch & Hemis. & Parabolo. & Cubic1D & Cubic2D & Saddle & Plane \\
\hline Cyl. & 48 & 6 & 20.5 & 2 & 1 & 3 & 6 & 11 \\
\hline Arch & & 55 & 3 & 13.5 & 4 & 7.5 & 10 & 2 \\
\hline Hemis. & & & 52 & 2.5 & 1 & 2 & 3 & 9 \\
\hline Parabolo. & & & & 68 & 6.5 & 4 & 5 & 1 \\
\hline Cubic1D & & & & & 74 & 13.5 & 3.5 & 1 \\
\hline Cubic2D & & & & & & 53 & 11 & 2 \\
\hline Saddle & & & & & & & 58 & 2.5 \\
\hline Plane & & & & & & & & 76 \\
\hline
\end{tabular}

aspect of our stimuli and the confusion matrices. Table 6 presents these correlations. In all of these correlations, the "correct" scores on the diagonal of the confusion matrix were transformed to their equivalent "error" scores by simply subtracting them from $100 \%$ prior to the computation. Table 6 also displays the Pearson correlation coefficients of the relationships among the two sets of stimuli $(r=.854)$, the correlations among the two sets of stimuli, and the confusion matrix results from all three of the experiments, as well as the correlations among the three sets of experimental results. The stimuli were measured in terms of the differences (in arbitrary units) between the range of depths of all pairings of the stimulus forms that define each cell in the confusion matrix. The results of this analysis can be summarized as follows.

1. The two sets of stimuli-those used in Experiment 1 and those used in Experiments 2 and 3, respectivelyare quite similar to each other $(r=.854)$ when compared in terms of the respective depth difference between corresponding cells in the two stimulus sets.

2. However, the stimuli, thus measured to reflect the maximum depth-difference aspect of the stimulus, correlate much less well with the three sets of experimental results than they do with themselves. The highest corre- lation between a stimulus set and performance is $r=$ -.715 , and the coefficients of correlation range down to as low as $r=-.576$. Furthermore, the stimuli used in Experiment 1, on the one hand, and in Experiments 2 and 3 , on the other, correlate no better with the appropriate experiments than with the other experiments in which they were not used. For example, the stimuli used in Experiments 2 and 3 correlate better with the performance of Experiment 1 than with that of Experiment $2(r=-.701$, as opposed to $r=-.666$ ). We now believe that this is mainly due to the fact that the two sets of stimuli were not actually very different from each other; they differed only in the extent to which the deep corners of the hemisphere and paraboloid of rotation were present in the second stimulus set but not in the first, and also in the size of the prototypes used to generate the two sets of stimulus forms. These differences were not influential in determining our observers' performance, mainly because of the low probability of a dot's falling in the peripheral areas of the first stimulus set.

3. The performance of the observers in the three experiments intercorrelate with each other in a way that also suggests that the difference between the two stimulus-form sets (used in Experiment 1 and in Experiments 2 and 3,

Table 5

The Grand Summary of the Folded and Reduced Confusion Matrices for All Three Experiments

\begin{tabular}{|c|c|c|c|c|c|c|c|c|}
\hline & Cyl. & Arch & Hemis. & Parabolo. & Cubic1D & Cubic2D & Saddle & Plane \\
\hline Cyl. & 53 & 6.2 & 16.2 & & & & 8 & 9.3 \\
\hline Arch & & 59 & & 10.6 & & & 10.1 & \\
\hline Hemis. & & & 60 & & & & & 10.6 \\
\hline Parabolo. & & & & 74 & & & & \\
\hline Cubic1D & & & & & 74 & 10 & & \\
\hline Cubic2D & & & & & & 60 & 9.6 & \\
\hline Saddle & & & & & & & 59 & \\
\hline Plane & & & & & & & & 74 \\
\hline
\end{tabular}


Table 6

The Set of Correlations Among the Stimuli, and the Results of Experiments 1, 2, and 3

\begin{tabular}{lrrrrr}
\hline & Stim. 1 & Stim. 2 & Exp. 1 & Exp. 2 & Exp. 3 \\
Stim. 1 & 1.000 & .854 & -.644 & -.575 & -.631 \\
Stim. 2 & & 1.000 & -.701 & -.666 & -.715 \\
Exp. 1 & & & 1.000 & .939 & .980 \\
Exp. 2 & & & & 1.000 & .967 \\
Exp. 3 & & & & & 1.000 \\
\hline
\end{tabular}

respectively) is not important. Experiment 3 correlates better with Experiment $1(r=.980)$ than it does with Experiment $2(r=.967)$, in spite of the fact that different stimulus sets were used. But the differences among these very high correlations are small, and the important conclusion is that the results of the three experiments are nearly identical in spite of the differences in stimulus forms that were used in each experiment.

Taken together, the pattern of these correlation coefficients indicates that only a modest amount of the variance in our observers' performance can be explained in terms of the relative range of depths of the surfaces, and that virtually none of the variance can be explained in terms of the difference between the two sets of stimulus forms. Therefore, we must look elsewhere for the stimulus attributes that created the particular pattern of confusions among the stimulus forms observed in these experiments.

Enclosed volume. Another possible dimension or attribute of the stimuli that was tested as a putative cause of the experimental results is the volume bounded by any two surfaces of the stimulus forms. This bounded volume is suggested as a global measure of the difference in shape of any two stimulus forms. Identical forms would thus produce a zero bounded volume; very dissimilar stimulus forms would, according to this argument, produce larger bounded volumes. Therefore, we carried out a similar correlation analysis comparing these bounded volumes and our performance data. Since no differential effect of the two stimulus-form sets had been obtained, this and all subsequent correlational analyses were carried out using only the second set-the set used in Experiments 2 and 3 -in the computations.

The correlations obtained between the bounded volumes and the results of the three experiments produce correlation coefficients of $-.761,-.788$, and -.776 , respectively. The results of this analysis show that this global factor is a better predictor of our experimental results than is the difference in depth ranges. However, only a little over half of the variance in our results is explained with this variable.

Area. We then tested the differences in surface areas (as calculated with a discrete approximation computational algorithm simulating a double integration) and found between these differences and performance scores correlations of $-.642,-.633$, and -.635 . These scores still leave the major amount of variance unexplained.

Slope. Next, we tested the ability of the slope difference measure $(\Delta S L)$ that approximated the first derivative of the surface (defined earlier in Equations 4 and 5) to predict the outcome of our three experiments. The correlation coefficients obtained in this case were -.767 , -.764 , and -.779 for the three experiments, respectively. While these values agree with the interexperimental correlations in being relatively stable, they also account for only about half of the variance of the experimental results. (Since the surface area and the measure of slope that we have used here are themselves very highly correlated $[r$ $=.92 \mathrm{]}$, it was not necessary to include both of these variables in subsequent analyses.)

We next considered a cumulative function of the second derivative of the stimulus-form surfaces. To accomplish this, we determined the second derivative $\left\{A\left[f_{n}\left(p_{m}\right)\right]\right\}$ of each point $\left(p_{m}\right)$ on each surface $\left(f_{n}\right)$ by evaluating the following expression:

$$
A\left[f_{n}\left(p_{m}\right)\right]=\sqrt{\left[\frac{\partial^{2} f_{n}\left(p_{m}\right)}{\partial y^{2}}\right]^{2}+\left[\frac{\partial^{2} f_{n}\left(p_{m}\right)}{\partial x^{2}}\right]^{2}+\left[\frac{\partial^{2} f_{n}\left(p_{m}\right)}{\partial x y}\right]^{2}}
$$

The following expression was then defined as a global

Table 7

The Summary of the Multiple Correlational Analysis

\begin{tabular}{lcccccc}
\hline & \multicolumn{7}{c}{$\mathrm{R}_{\mathrm{s}}$} \\
\cline { 2 - 6 }$\Delta$ Exp. 1 & Exp. 2 & Exp. 3 & $\Delta$ Depth & $\Delta$ Slopes & $\Delta$ Volumes \\
$\Delta$ Depth & -.7010 & -.6693 & -.7174 & 1.0000 & .7509 & .4270 \\
$\Delta$ Slopes & -.7669 & -.7641 & -.7793 & .7509 & 1.0000 & .6987 \\
$\Delta$ Volumes & -.7630 & -.7868 & -.7750 & .4270 & .6987 & 1.0000
\end{tabular}

\begin{tabular}{lccc} 
& Exp. 1 & Exp. 2 & Exp.3 \\
Beta1 & -.4035 & -.3444 & -.4220 \\
Beta2 & -.0100 & -.1143 & -.0915 \\
Beta3 & -.5208 & -.5599 & -.5309 \\
\hline
\end{tabular}


figure of merit $(\Delta A)$ of the differences between all of the points on the second derivatives of all pairs of stimulus forms:

$$
\Delta A=\Sigma\left|A\left[f^{1}\left(p_{m}\right)\right]-A\left[f^{2}\left(p_{m}\right)\right]\right|
$$

When the second derivative differences were correlated, our experimental results-correlation coefficients of $-.760,-.761$, and -.777 -were obtained for Experiments 1,2 , and 3 , respectively. These numbers are very close to those resulting from the analysis of the relationship between our results and the first derivative-as they should be given the close relationship between these two transformations-and therefore will not be considered further.

Probability of distribution. Looking at the problem from another point of view, we computed the probability that the dots sampling a particular stimulus form would fall into one of eight equal-depth segments. We computed the probability that a dot would fall into each of these segments and calculated the probability of selecting a point from the same segment for each possible pair of stimulus forms. This measure was suggested by the hypothesis that confusions may occur if sample dots from two different stimulus forms are likely to be at the same depths. Unfortunately, this hypothesis also had to be rejected, since the correlations between this probability distribution and the performance scores for the three experiments were $.408, .617$, and .488 , respectively, for the three experiments.

Multiple cue determination. Therefore, no single attribute of the stimulus forms used in this study seemed to account for much more than half of the variance and to produce the desired predictability. We therefore suggest that our observers' judgments were based on several of these stimulus-form factors, rather than on a single factor. To test this hypothesis, we carried out a multiple correlation analysis in an attempt to achieve a better accounting of the cues that might account for the performance of our observers. The best prediction of performance scores was produced by a multiple correlation in which the second of the stimulus-form geometries, the one used in Experiments 2 and 3, was combined with the difference-of-slopes metric $(\Delta S L)$ and the measures of the bounded volume enclosed between the two curves. These three parameters are relatively weakly correlated with each other (as shown in the summary of the multiple correlation in Table 7) and are therefore not as redundant with each other as some of the other variables. Collectively, however, using the multiple correlation approach, they did predict performance in the three experiments, with multiple correlation coefficients of $.829, .870$, and .886 for Experiments 1, 2, and 3, respectively. At this point we are accounting for $68.7 \%, 75.6 \%$, and $78.4 \%$ of the variance in Experiments 1, 2, and 3, respectively.

It is important to point out that each of the three measures we used in this multiple correlation analysis is likely to cross-correlate with other measures, some that we have tested and others that we have not. Clearly, a surface with the same two-dimensional projection as another but with a larger surface area would tend to have a larger $\Delta S L$ than the other. (However, because of the way the $\Delta \mathrm{SL}$ was defined, this is not necessarily true.) Similarly, the derivative (or some arbitrary figure of merit, such as $\Delta \mathrm{A}$ ) of the equation for a particular shape must also be closely related to the equation of the shape itself.

There are two important results implicit in this analysis. The first is that no single attribute of the set of stimulus forms that we used is sufficient to account for the obtained performance scores. Rather, as more and more attributes are involved in the multiple correlation, the predictions progressively improve. The second important conclusion is that the best predictors are those-the bounded volume and $\Delta \mathrm{SL}$ (and others closely related to them) - that are global estimates of the shapes of the surfaces. We have determined, therefore, that the observers in this study were more strongly cued by the overall shapes of the surfaces than by any of the local features that might have been present in the stimulus.

\section{CONCLUSION}

We believe that in this study we have demonstrated one particular and possible composite set of cues that predicts fairly well the recognizability of a particular set of nonplanar surfaces. The fact that no single attribute could be identified that would enable the observer to recognize the shapes of our stimulus surfaces is a compelling argument for two conclusions.

1. Shape recognition of the kind exemplified by the task used in the present study is a global process in which the general arrangement of the parts of the stimulus are more important than the nature of the parts themselves.

2. Observers in this task utilized a multidimensional set of cues in order to perform at the very high levels of reconstruction that we measured. Our observers were able to reconstruct (as evidenced by their extraordinary success at recognizing these sparsely sampled forms) the general shape of the nonplanar surfaces from exceedingly sparse samples. They seem to have done so not on the basis of any single cue, but rather on the basis of multiple aspects of the stimulus, which collectively define the "shape of the surface." Of course, we cannot exclude the possible presence of a single critical cue that may have correlated highly with the best predicting combination, but we were not able to identify it if it did, in fact, exist.

Another important outcome of this study is that our observers performed better than a first approximation to a model for the reconstruction process suggests that they should. The simple polynomial, surface-fitting procedure, which we used as an anchoring reference, requires at least 5 dots to reconstruct a quadric and 7 dots to reconstruct a cubic surface. (We have confirmed this in a computational simulation-no shape other than a plane was graphically reconstructed with fewer than these minima.) Obviously there must have been other cues and constraints built into our sampled stimulus set that provided our ob- 
servers with the information that enabled them to do better than the surface-fitting algorithm. We have examined some of the cues and constraints here, but obviously not all those possible. The task that we presented to our observers is obviously not the same one presented to the model.

Finally, although it is impossible to be absolutely conclusive, we believe that this pattern of results and analyses argues strongly for a globally precedent, and probably parallel, processing algorithm for this kind of three-dimensional visual information task. In light of these data, and in the absence of any local "features" of any kind in these dotted stimuli, it is difficult to imagine how any theory oriented toward feature processing could account for these data. Nevertheless, the development of a complete global and parallel model for this powerful human form-perception process is a task yet to be achieved.

\section{REFERENCES}

Bachmann, T. (1978). Cognitive contours: Overview and a preliminary theory. Acta et Commentationes Universitatis Tartuensis \#474. Problems of Communication \& Perception (31-59). Tartu, Estonia: Tartu State University.

Bachmann, T. (1980). Genesis of subjective image. Acta et Commentationes Universitatis Tartensis \#522. Problems of Cognitive Psychology, 102-126.

Braunstein, M. L. (1988). The empirical study of structure from motion. In W. N. Martin \& J. K. Aggarwal (Eds.), Motion understanding: Robot and human vision. Hingham, MA: Kluwer Academic Publishers.

GrImSON, W. E. L. (1981). From images to surfaces: A computational study of the human early visual system. Cambridge, MA: MIT Press.

Hilbert, D., \& Cohn-Vossen, D. (1952). Geometry and the imagination. New York: Chelsea.

HoRN, B. K. P. (1975). Obtaining shape from shading information. In P. H. Winston (Ed.), The psychology of computer vision (pp. 115155). New York: McGraw-Hill.

JuLEsZ, B. (1960). Binocular depth perception of computer generated patterns. Bell System Technical Journal, 39, 1125-1162.

Julesz, B. (1971). Foundations of cyclopean perception. Chicago: University of Chicago Press.
Kanizsa, G. (1974). Contours without gradients or cognitive contours. Italian Journal of Psychology, 1, 93-112.

KOHLER, W. (1947). Gestalt psychology: An introduction to the new concepts in modern psychology. New York: Liveright. (Original work published 1929)

LaPPin, J. S., Doner, J. F., \& Kotas, B. L. (1980). Minimal conditions for the visual detection of structure and motion in threedimensions. Science, 209, 717-719.

LAPPIN, J. S., \& FuQUA, M. A. (1983). Accurate visual measurement of three-dimensional moving patterns. Science, 221, 480-482.

MARR, D. (1982). Vision: A computational investigation into the human representation and processing of visual information. San Francisco: W. H. Freeman.

Petry, S., \& Meyer, G. E. (1986). Adelphi International Conference on Illusory Contours: A report on the conference. Perception \& Psychophysics, 39, 210.

STEVENS, K. A. (1986). Inferring shape from contours across surfaces. In A. P. Pentland (Ed.), From pixels to predicates: Recent advances in computational vision (pp. 93-110). Norwood, NJ: Ablex.

Townsend, J. T., \& AshBY, F. G. (1982). Experimental test of contemporary mathematical models of visual letter recognition. Journal of Experimental Psychology: Human Perception \& Performance, 8 , 834-864.

UlLMAN, S. (1979). The interpretation of visual motion. Cambridge, MA: MIT Press.

UTTAL, W. R. (1987). The perception of dotted forms. Hillsdale, NJ: Erlbaum.

WERTHEIMER, M. (1923). Unterchungen zur Lehre von der Gestalt, II. Psychologische Forschung, 4, 301-350.

\section{NOTE}

1. Such a strategy also has the very important ancillary effect of removing our research from the domain of "How do observers solve the dichoptic correspondence problem?" and placing it in the domain of "How do observers recognize and reconstruct stereoscopic images?" The latter question defines the domain of research of this study; it is important that it be distinguished from the questions asked by those interested in the mechanisms and processes underlying the registration of disparate images on corresponding retinal points. Throughout the study, we sought to irreducibly minimize the difficulty of the registration aspects of the task so that we could study the processing of the perceived depth information, not its generation from disparate images.

(Manuscript received April 13, 1987; revision accepted for publication June 30, 1987.) 